\title{
ON THE CHARACTERISATION OF POLAR FIBROUS COMPOSITES WHEN FIBRES RESIST BENDING - PART III: THE SPHERICAL PART OF THE COUPLE-STRESS
}

\author{
K.P. Soldatos \\ School of Mathematical Sciences, University of Nottingham, Nottingham NG7 2RD, UK
}

\begin{abstract}
Part II (Soldatos 2018b) identified some theoretical disagreement between the generally anisotropic polar linear elasticity of Mindlin and Tiersten (1962) and its counterpart developed in (Spencer and Soldatos, 2007; Soldatos, 2014) for fibrous composites with embedded fibres resistant in bending. The present communication shows that this disagreement is essentially due to inherent features of fibre-splay types of deformation and, consequently, generalises the couple-stress theory in a manner that creates room for newly emerged fibre-splay type of kinematic variables to enter and be accounted for. Relevant fundamental theorems, associate in Part II with the Mindlin and Tiersten model, are generalised accordingly to meet the needs and requirements of the proposed new formulation. Interestingly and importantly, the outlined generalisation enables formation of a convincing answer to a long-standing question regarding the indeterminacy of the spherical part of the couple-stress tensor, at least as far as polar elasticity of fibre-reinforced materials is concerned. The manner thus is demonstrated in which the spherical part of the couplestress can be determined in polar linear elasticity of fibrous composites that exhibit transverse isotropy due to an embedded family of fibres resistant in bending.
\end{abstract}

Keywords: Couple-stress theory, Elasticity, Fibre-reinforced materials, Fibres resistant in bending, Linear elasticity, Polar elasticity, Spherical part of couple-stress. 


\section{Introduction}

Part II (Soldatos 2018b) presented an initial theoretical comparison of the anisotropic version of, and principal postulations stemming from the linear polar elasticity due to Mindlin and Tiersten (1962) with their counterparts presented in (Spencer and Soldatos, 2007; Soldatos, 2014, 2015) for linear elasticity modelling of fibrous composites with embedded fibres that resist bending. The performed comparison confined attention to potential solutions of relevant boundary value problems described by continuous displacements that possess continuous derivatives of all orders (for simplicity referred to as "continuous solutions"), and was underpinned by the common theoretical background that those models share with the Cosserat couple-stress theory (Cosserat and Cosserat, 1909).

It is thus recalled that, due to their different origin, scope and formulation, the compared theoretical formalisms fail, in general, to agree completely. Their difference was rather marginal in the case of unidirectional fibrous composites modelled with the restricted version of the Spencer and Soldatos (2007) formalism (see also (Soldatos, 2014)) that accounts for the fibre-bending deformation only. However, it became evident when the unrestricted theory that accounts for the known coupling of all three, fibre-bending, fibresplay and fibre-twist deformation modes was encountered. Part II made no attempt to identify the origin of, or to fix the observed theoretical disagreement which, however, motivated subsequent studies that lead to the present communication.

It will accordingly be shown in this communication, that the implied disagreement is essentially due to inherent features of fibre-splay types of deformation. As was pointed out in Part II, those features are manifested as new kinematic variables (Spencer and Soldatos, 2007; Soldatos, 2014), and are seemingly inflicted by second-gradient deformation effects that represent gradients of the strain field along the fibres. These thus are neither expressible in terms of the strains nor in terms of the spin-gradient kinematic variables employed in conventional Cosserat and Cosserat (1909) elasticity, including its relevant Mindlin and Tiersten (1962) formulation. Nevertheless, Soldatos et al. (2020) have now introduced a second restricted version of the linear theory presented in (Spencer and Soldatos, 2007; Soldatos, 2014) which distinguishes the most predominant set of the 
implied fibre-splay features and, hence, enables their study in isolation from their fibrebending and fibre-twist counterparts.

Under these considerations, Section 2 begins by reiterating the common Cosserat and Cosserat (1909) theoretical background shared by the Spencer and Soldatos (2007) polar elasticity formalism and the generally anisotropic polar linear elasticity model due to Mindlin and Tiersten (1962), and, in this manner, also serves as a proper reminder of the principal equations met in conventional couple-stress theory. Moreover, it identifies the reason responsible for the outlined theoretical disagreement and, consequently, postulates a generalised formulation of the couple-stress theory that creates room for those newly emerged fibre-splay type of kinematic variables to enter and be accounted for. Section 3 then completes the presented formulation by providing appropriate generalisations of relevant fundamental theorems associated in Part II with the Mindlin and Tiersten model.

Most importantly, the results of the presented generalisation enable Section 4 to form and provide a convincing answer to a long-standing question regarding the indeterminacy of the spherical part of the couple-stress tensor, at least as far as polar elasticity of fibre-reinforced materials is concerned. It is recalled that this well-known indeterminacy has become a subject of wonder, discussion and debate for many decades (e.g., Mindlin and Tiersten, 1962; Koiter, 1964; Eringen, 1968; Spencer and Soldatos, 2007; Hadjesfandiari and Dargush, 2011; Soldatos, 2014).

Section 4 thus provides an initial connection between the newly established generalised formulation and the polar elasticity models studied in Part II. Moreover, it provides additional means and an extra piece of information that enable the generalised couple-stress theory developed previously in Section 2 to fully determine the couple-stress tensor, including its spherical part. In doing so, it requires from that extra piece of information, to suitably accommodate the an extra energy term (Spencer and Soldatos, 2007; Soldatos, 2014) that offers no contribution to the constitutive equations and, like the spherical part of the couple-stress, does not influence the state of equilibrium.

Section 5 then examines in detail the impact that this connection has on each one of the three available versions of polar linear elasticity for fibrous composites that exhibit transverse isotropy due to an embedded family of fibres resistant in bending. A summary of 
the most important conclusions thus made is presented in Section 6, where directions are also provided for possible future relevant developments.

\section{Generalised formulation of the couple-stress theory for linearly elastic solids}

\subsection{Conventional couple-stress theory}

As is also noted in Truesdell and Toupin (1960), Mindlin and Tiersten (1962), as well as in Part II and elsewhere, description of the principal equations of the Cosserats (1909) couplestress theory may begin with the standard decomposition,

$\sigma_{i j}=\sigma_{(i j)}+\sigma_{[i j]}$,

of the non-symmetric stress tensor, $\boldsymbol{\sigma}$, into its symmetric and antisymmetric parts, and continue with the equilibrium equations

$$
\sigma_{i j, i}=0, \quad \sigma_{[i j]}=\frac{1}{2} \varepsilon_{k j i} m_{\ell k, \ell},
$$

where $\boldsymbol{m}$ denotes the corresponding couple-stress tensor. Here, as well as in what follows, indices refer to some suitable Cartesian co-ordinate framework, $\mathrm{O} x_{i}$, and take the values 1,2 and 3 (the summation notation of repeated indices also applies). Moreover, body forces and body couples are neglected for simplicity, while the indicated components of the tensors $\sigma$ and $\boldsymbol{m}$ are all assumed differentiable functions of the implied co-ordinate parameters.

Under the assumption that not only the components of $\boldsymbol{m}$, but also their derivatives appearing in (2.2b) are differentiable, a combination of (2.1) with the equilibrium equations (2.2) leads to

$$
\sigma_{(i j), i}+\frac{1}{2} \varepsilon_{k j i} \bar{m}_{\ell k, \ell i}=0 \text {, }
$$

where

$$
\bar{m}_{\ell k}=m_{\ell k}-\frac{1}{3} m_{r r} \delta_{\ell k}
$$

is the deviatoric part of the couple-stress tensor, and the appearing Kronecker's delta represents the components of the unit matrix, $\boldsymbol{I}$. No constitutive equations are provided or required at this point. It is recalled though that the evident absence of the spherical part, 
$m_{r r}$, from the equations of equilibrium (2.3) has underpinned for long time the feeling that this is generally indeterminate in polar elasticity (e.g., Truesdell and Toupin, 1960; Mindlin and Tiersten, 1962; Koiter, 1964; Mindlin and Eshel, 1968; Eringen, 1968; Spencer and Soldatos, 2007; Hadjesfandiari and Dargush, 2011; Soldatos, 2014).

Along with the above equations, the components of the traction and the coupletraction vectors acting on any internal or bounding surface of the material are respectively given as follows:

$T_{i}^{(n)}=\sigma_{j i} n_{j}, \quad L_{i}^{(n)}=m_{j i} n_{j}$,

where $n$ denotes the outward unit normal of that surface. In the case of the bounding surface of an elastic solid, (2.5) represent the traction and couple-traction boundary conditions applied externally on the material, respectively.

\subsection{Polar material behaviour of fibrous composites}

It is initially recalled that the infinitesimal rotation field $\omega$, which is the antisymmetric part of the displacement gradient (see (2.11) below, and Part II), is generally present always in a deformation, regardless of whether the fibrous composite of interest exhibits polar or nonpolar material behaviour. In the latter case though, which embraces conventional linear elasticity, $\boldsymbol{\omega}$ does not produce rotation energy. This is due to the symmetry of the stress field or, equivalently, due to the subsequent absence of the antisymmetric part of that field.

It is now noted that polar material behaviour due to the bending resistance of individual fibres can take place in the absence of externally applied couple-stress tractions. It is accordingly clear that when $\boldsymbol{L}^{(n)}=\mathbf{0}$ on the external boundary, couple-stress is generated internally, as soon as fibres with bending stiffness deform, and their direction gradients thus enable them to act within the material as embedded Euler-Bernoulli beams. As is implied by (2.2b), the emerging couple-stress field gives then rise to the antisymmetric part of the stress which, in turn, interacts with the rotation field, $\boldsymbol{\omega}$, and creates work that is stored as rotation energy in the deformed material.

These observations suggest that there is generally no apparent reason for the general rotation field of the deformation, $\boldsymbol{\omega}$, to coincide with the specific fibre rotation field, $\boldsymbol{\varphi}$ say, that characterises the polar material behaviour of a fibrous composite. It follows that the 
spin-vectors $\boldsymbol{\Omega}$ and $\boldsymbol{\Phi}$ that correspond to the different rotation fields $\boldsymbol{\omega}$ and $\boldsymbol{\varphi}$, respectively, do not coincide either.

Under these considerations, a generalised formulation of the Cosserats' couplestress theory can be established by (i) replacing in the energy/power balance equation (2.8) of Part II the appearing time derivative of the spin-vector, $\dot{\Omega}_{i}$, with its $\dot{\Phi}_{i}$ counterpart, and (ii) following afterwards identical steps to those detailed there. Nevertheless, the linear character of the formulation endorses here promotion of an alternative, slightly more direct approach that enables a priori validity of the Clapeyron's theorem extension presented in Part II.

It is pointed out that the present formulation is motivated by its usefulness in polar elasticity of fibre-reinforced materials. However, it may potentially be found useful in other, possible polar elasticity branches in which the characteristic spin-vector $\boldsymbol{\Phi}$ acquires some different meaning, and thus is not necessarily related to fibre deformation.

\subsection{Generalised couple-stress theory of linearly elastic solids}

In line with the polar material extension of Clapeyron's theorem (Theorem 1 in Part II), the present generalised formulation begins by postulating that, in the absence of body forces and body moments, the total energy stored within an arbitrary volume, $V$, of the polar linearly elastic material of interest is

$$
E=\frac{1}{2} \int_{S}\left(T_{i}^{(n)} u_{i}+L_{i}^{(n)} \Phi_{i}\right) d S \geq 0,
$$

where $S$ denotes the surface that surrounds $V, d S$ represents the corresponding surface element, and the equality sign holds only in the absence of deformation. Moreover, $\boldsymbol{u}$ (with components $u_{i}$ ) is the standard displacement vector that is conjugate to the traction vector $\boldsymbol{T}^{(n)}$, and $\boldsymbol{\Phi}$ (with components $\Phi_{i}$ ) is some appropriately defined/specified spin-type vector, which (i) characterises the couple-stress theory of interest, (ii) is generally dependent of the displacement gradients, and (iii) is conjugate to the couple-traction vector $\boldsymbol{L}^{(n)}$. Such a characteristic spin-type vector, $\boldsymbol{\Phi}$, is perceived as the vector of a corresponding, antisymmetric, rotation-type tensor, $\varphi$, in the sense that 
$\Phi_{i}=\frac{1}{2} \varepsilon_{i j k} \varphi_{k j}, \quad \varphi_{k j}=\varepsilon_{i j k} \Phi_{i}$.

Regardless of the choice of $\boldsymbol{\Phi}$, a combination of (2.5) and (2.6), followed by application of the divergence theorem and the product rule of differentiation, leads to

$E=\frac{1}{2} \int_{V}\left[\sigma_{j i} u_{i, j}+\left(m_{j i} \Phi_{i}\right)_{, j}\right] d V$

where (2.2a) is also accounted for. Moreover, due to the symmetry and the antisymmetry, respectively, of the standard small strain and rotation tensors,

$e_{i j}=\frac{1}{2}\left(u_{i, j}+u_{j, i}\right), \omega_{i j}=\frac{1}{2}\left(u_{i, j}-u_{j, i}\right)$,

(2.8) is seen equivalent to the following:

$E=\frac{1}{2} \int_{V}\left[\sigma_{(j i)} e_{i j}+\sigma_{[j i]} \omega_{i j}+\left(m_{\ell i} \Phi_{i}\right)_{, \ell}\right] d V$.

Use of (2.2b) then leads to

$E=\frac{1}{2} \int_{V}\left[\sigma_{(j i)} e_{i j}+\frac{1}{2} \varepsilon_{k i j} m_{\ell k, \ell} \omega_{i j}+\left(m_{\ell i} \Phi_{i}\right)_{, \ell}\right] d V$,

and through use of the first of the well-known relationships

$\Omega_{\mathrm{i}}=\frac{1}{2} \varepsilon_{i j k} \omega_{k j}, \omega_{k j}=\varepsilon_{i j k} \Omega_{i}$,

one finally obtains

$E=\frac{1}{2} \int_{V}\left[\sigma_{(j i)} e_{i j}+m_{\ell i, \ell}\left(\Phi_{i}-\Omega_{i}\right)+m_{\ell i} \Phi_{i, \ell}\right] d V \equiv \int_{V}\left(W^{e}+W^{\Phi}\right) d V=\int_{V} W d V$

It is recalled that the conventional/general spin-vector (2.11a) is the vector of the antisymmetric rotation tensor (2.11b). Moreover, the positive semidefinite quantities

$W^{e}=\frac{1}{2} \sigma_{(j i)} e_{i j} \geq 0, \quad W^{\Phi}=\frac{1}{2}\left[m_{\ell i, \ell}\left(\Phi_{i}-\Omega_{i}\right)+m_{\ell i} \Phi_{i, \ell}\right] \geq 0$,

represent the standard strain energy function met in nonpolar linear elasticity and a corresponding generalised spin-gradient counterpart which is due to the observed polar response of the material. 
It is pointed out that the equality sign appearing in (2.13a) or (2.13b) applies only in the absence of strain or polar material response, respectively. In this manner, the internal energy stored in the material is guaranteed to also be positive semidefinite,

$W \geq 0$,

with the equality holding only in the complete absence of deformation.

The outlined generalised formulation of the couple-stress theory will be connected in Section 4 with the anisotropic, polar, linear elasticity models considered and discussed in Part II. It will thus be revealed that, when couple-stress creation is specifically due to fibre bending resistance, a mechanism does exist that enables determination of the spherical part of couple-stress tensor. This observation can accordingly lead to a positive conclusion of the long-standing debate regarding the relevant well-known weakness of the conventional formulation (Cosserat and Cosserat, 1909). Meanwhile though, Section 3 demonstrates the manner that a generalisation becomes possible of the fundamental theorems associated in Part II with the anisotropic version of the polar linear elasticity model due to Mindlin and Tiersten (1962).

\section{Generalisation of associated fundamental theorems}

Part II proved the validity of five fundamental theorems that hold true in association with the Cosserat and Cosserat (1909) polar elasticity formalism and its generally anisotropic linear version due to Mindlin and Tiersten (1962). The first of them (Theorem 1 in Part II) consists of a polar material extension of Clapeyron's theorem, and a comparison of (2.6) with (2.12) confirms that its validity is already incorporated into the present formulation. Indeed, this comparison shows that "the sum of the total strain and spin-gradient energies of deformation equals one half of the work done by the external forces and moments acting through their ultimate displacement and generalised spin-vector fields, $\boldsymbol{u}$ and $\boldsymbol{\Phi}$, respectively”.

Nevertheless, $(2.13 \mathrm{~b})$ reveals further that

$$
W^{\Phi}=\frac{1}{2}\left[m_{\ell i, \ell}\left(\Phi_{i}-\Omega_{i}\right)+m_{\ell i} \Phi_{i, \ell}\right]=\frac{1}{2}\left[\left(m_{\ell i} \Phi_{i}\right)_{, \ell}-m_{\ell i, \ell} \Omega_{i}\right]
$$

As the rotation energy introduced in Part II is 
$W^{\omega}\left(\omega_{i j}\right)=\frac{1}{2} \sigma_{[j i]} \omega_{i j}=\frac{1}{4} \varepsilon_{k i j} m_{\ell k, \ell} \omega_{i j}=-\frac{1}{2} m_{\ell k, \ell} \Omega_{k}$,

it follows that

$$
W^{\Phi}-W^{\omega}=\frac{1}{2}\left(m_{\ell i} \Phi_{i}\right)_{, \ell}
$$

This is clearly a generalisation of the corresponding relationship (3.11) of Part II, which is obtained by here replacing $W^{\Phi}$ and $\boldsymbol{\Phi}$ with $W^{\Omega}$ and $\boldsymbol{\Omega}$, respectively.

Integration of (3.3) over the volume element $V$ leads to

$$
\int_{V}\left(W^{\Phi}-W^{\omega}\right) d V=\frac{1}{2} \int_{V}\left(m_{\ell i} \Phi_{i}\right)_{, \ell} d V=\frac{1}{2} \int_{S} m_{\ell i} \Phi_{i} n_{\ell} d S=\frac{1}{2} \int_{S} L_{i}^{(n)} \Phi_{i} d S,
$$

where use is also made of the divergence theorem and (2.5b). A combination of (3.4) with (2.6) and (2.12) then yields

$$
\int_{V}\left[W^{e}\left(e_{i j}\right)+W^{\omega}\left(\omega_{i j}\right)\right] d V=\frac{1}{2} \int_{S} T_{i}^{(n)} u_{i} d S,
$$

which is identical with what is presented in Part II as the alternative form of

\section{Theorem 1:}

If a polar linearly elastic body of volume $V$ is in equilibrium under the action of tractions $\boldsymbol{T}$ and couple-tractions $\boldsymbol{L}$ applied externally on its bounding surface $S$, then the sum of the total strain and rotation energies of deformation equals one half of the work done by the external forces acting through their ultimate displacement field, $\boldsymbol{u}$.

It is thus concluded that in this "alternative form", the polar material extension of Clapeyron's theorem acquires a generalised status which is independent of the spin-type vector, $\boldsymbol{\Phi}$, that characterises the couple-stress theory of present interest.

Validity of (3.2) shows next that Theorem 2 of Part II can acquire a slightly more general form. Indeed, by setting $\boldsymbol{L}^{(n)}=\mathbf{0}$ into the right-hand-side of (3.4), it is immediately seen that

\section{Theorem 2:}


If a polar linearly elastic body is in equilibrium under the action of homogeneous coupletraction boundary conditions $\left(\boldsymbol{L}^{(n)}=\mathbf{0}\right)$, then the total rotation energy stored in its material equals its generalised spin-gradient counterpart.

The third fundamental theorem presented in Part II can also be generalised in a similar manner, and obtain the following form:

\section{Theorem 3:}

In the generalised couple-stress theory of linear elasticity, a well-posed boundary value problem can have only a single continuous solution.

It is briefly recalled in this regard that the term "continuous solution" distinguishes in Part II, as well as in here, a solution described by continuous displacements that possess continuous derivatives of all orders, from any of its potential "weak discontinuity" counterparts; see also (Soldatos et al, 2020). In Part II, the proof of that theorem was provided in an Appendix. That proof can be extended and thus seen applicable in the present generalised case by just replacing the symbol $\Omega$ with a symbol $\Phi$, wherever $\Omega$ appears in Appendix B of Part II. Such a repetition is felt unnecessary here.

The polar material extension of the theorem of minimum potential energy provided in Part II (Theorem 4) is also valid here, subject to minimal modifications. Its proof is essentially identical to its counterpart detailed in Part II, thus leading to

Theorem 4 (Theorem of minimum potential energy in generalised couple-stress linear elasticity):

Of all continuous and differentiable displacement fields $\boldsymbol{u}^{*}$ which (i) satisfy the displacement boundary conditions on $S^{u}$, and (ii) possess up to third order continuous and differentiable derivatives, the field $\boldsymbol{u}$ that represents the single continuous solution of a well-posed boundary value problem within the present, generalised framework of couple-stress linear elasticity yields a minimum value of the potential energy functional

$$
P\left(u_{i}\right)=\int_{V}\left[W^{e}\left(e_{i j}\right)+2 W^{\omega}\left(\omega_{i j}\right)\right] d V-\int_{S^{T}} T_{i}^{B} u_{i} d S
$$


which, by virtue of (3.4), is equivalent to

$$
P\left(u_{i}\right)=\int_{V}\left(W^{e}+2 W^{\Phi}\right) d V-\int_{S^{T}}\left(T_{i}^{B} u_{i}+L_{i}^{B} \Phi_{i}\right) d S .
$$

Here $S^{T}$ represents the part of the outer surface $S$ that boundary tractions, $T_{i}^{B}$, and coupletractions, $L_{i}^{B}$, are prescribed on. The remaining part of the bounding surface, where boundary displacements, $u_{i}^{B}$, and boundary spins, $\Phi_{i}^{B}$, are prescribed on, is denoted by $S^{u}$, so that $S=S^{u} \cup S^{T}$.

In part II, Theorem 5 is already associated with any well-posed linear elasticity boundary value problem in which $W^{\omega}=0$. In this context, the theorem also holds unchanged in the present formulation, though it is here repeated for the sake of selfsufficiency. Accordingly,

\section{Theorem 5:}

In linear elasticity, a well-posed mixed boundary value problem that stores no rotation energy is free from weak discontinuity solutions and, therefore, possesses a unique continuous solution.

The consequences of this theorem are also held unchanged in the present case and, as they are detailed in Section 3 of Part II, they are not repeated here. Nevertheless, by setting in

$W^{\omega}=0$,

one obtains

$$
\int_{V} W^{\Phi} d V=\frac{1}{2} \int_{S} L_{i}^{(n)} \Phi_{i} d S,
$$

which serves here as generalisation of the corresponding relationship (3.22) obtained in Part II.

\section{Connection with the specific couple-stress models considered in Part II}


4.1 The Cosserat and Cosserat (1909) couple-stress theory, and the Mindlin and Tiersten (1962) model for generally anisotropic, polar, linearly elastic solids

The outlined generalised polar elasticity formulation reduces to its Cosserat and Cosserat (1909) counterpart as soon as

$\Phi_{i} \equiv \Omega_{i}$,

and the characteristic spin-vector $\boldsymbol{\Phi}$ is thus assumed identical to its standard counterpart defined in (2.11a). In that case, expressions (2.13) reduce to

$$
W^{e}=\frac{1}{2} \sigma_{(j i)} e_{i j}, \quad W^{\Phi} \equiv W^{\Omega}=\frac{1}{2} m_{\ell i} \Omega_{i, \ell}=\frac{1}{2} \bar{m}_{\ell i} \Omega_{i, \ell} .
$$

The Mindlin and Tiersten (1962) model becomes thus available as soon as these energy expressions are associated with the relevant, specific set of constitutive equations employed in (Mindlin and Tiersten, 1962); see also Part II (Soldatos, 2018b).

Consequently, each one of the theorems detailed in the preceding Section reduces naturally into the form of its counterpart presented in Section 3 of Part II. It is recalled that (4.2a) implies that the form of the constitutive equation for the symmetric part of stress tensor coincides with its well-known non-polar elasticity counterpart (e.g., Ting, 1996; Jones, 1998).

Expressions (4.2) thus verify that the spherical part of the couple-stress tensor, $m_{r r}$, neither enters the relevant constitutive equations nor the equations of equilibrium (2.3) and, hence, does not influence the state of equilibrium. On the other hand, the energy functional (2.12) reduces to

$$
E=\frac{1}{2} \int_{V}\left[\sigma_{(j i)} e_{i j}+\bar{m}_{\ell i} \Omega_{i, \ell}\right] d V \equiv \int_{V}\left(W^{e}+W^{\Omega}\right) d V=\int_{V} W d V
$$

where use is made of (2.4), along with the identity $\Omega_{i, i}=0$. As $m_{r r}$ does not contribute into the stored energy either, it is naturally left indeterminate in the Mindlin and Tiersten model.

\subsection{Polar linear elasticity for fibre-reinforced solids}


In the Spencer and Soldatos (2007) linear elasticity framework (see also Soldatos, 2014), polar material behaviour is associated with transverse isotropy due to the presence of a single family of fibres and, as is justified in Section 2.2, it anticipated that $\Phi_{i} \neq \Omega_{i}$.

As the polar material effects encountered in the present framework are due to the deformation of the fibre direction vector, $\boldsymbol{a}$, it is reasonable for someone to relate the fibre spin-vector, $\boldsymbol{\Phi}$, that characterises the present theory with the vector product

$\tilde{\boldsymbol{\Phi}}=\boldsymbol{a} \times(\boldsymbol{b}-\boldsymbol{a})=\boldsymbol{a} \times \boldsymbol{b}$,

where the components of the deformed fibre vector, $\boldsymbol{b}$, are

$b_{i}=F_{i j} a_{j}=\left(\delta_{i j}+u_{i, j}\right) a_{j}=a_{i}+u_{i, j} a_{j}$,

and $\boldsymbol{F}$ represents the deformation gradient tensor.

It is accordingly postulated that, in components, the fibre spin-vector, $\boldsymbol{\Phi}$, is of the form

$$
\Phi_{i}=\tilde{\phi} \tilde{\Phi}_{i}=\tilde{\phi} \varepsilon_{i j k} a_{j}\left(b_{k}-a_{k}\right)=\tilde{\phi} \varepsilon_{i j k} u_{k, \ell} a_{\ell} a_{j},
$$

where the scalar parameter $\tilde{\phi}$ is to be determined and is generally anticipated dependent on the deformation. Connection of this definition with $(2.7 b)$ gives the components of the corresponding characteristic fibre-rotation tensor as follows:

$$
\varphi_{k j}=\frac{\tilde{\phi}}{2} \varepsilon_{i j k} \varepsilon_{i \ell m} a_{\ell} b_{m}=\frac{\tilde{\phi}}{2}\left(\delta_{j \ell} \delta_{k m}-\delta_{j m} \delta_{k \ell}\right) a_{\ell} b_{m}=\frac{\tilde{\phi}}{2}\left(a_{j} b_{k}-a_{k} b_{j}\right)=\frac{\tilde{\phi}}{2}\left(a_{j} u_{k, \ell}-a_{k} u_{j, \ell}\right) a_{\ell} .
$$

The generalized spin energy $(2.13 \mathrm{~b})$ can then be rearranged and take the form

$$
\begin{aligned}
W^{\Phi}=\frac{1}{2}\left[\left(\frac{1}{3} m_{r r, \ell} \delta_{\ell i}+\bar{m}_{\ell i, \ell}\right)\left(\tilde{\phi} \tilde{\Phi}_{i}-\Omega_{i}\right)+\left(\frac{1}{3} m_{r r} \delta_{\ell i}\right.\right. & \left.\left.\bar{m}_{\ell i}\right)\left(\tilde{\phi} \tilde{\Phi}_{i}\right)_{, \ell}\right] \\
& =W_{1}^{\Phi}\left(m_{r r}, \tilde{\phi}\right)+W_{2}^{\Phi}\left(\bar{m}_{\ell i}, \tilde{\phi}\right),
\end{aligned}
$$

where

$$
\begin{aligned}
& W_{1}^{\Phi}\left(m_{r r}, \tilde{\phi}\right)=\frac{1}{6}\left[\left(m_{r r} \tilde{\phi} \tilde{\Phi}_{i}\right)_{, i}-m_{r r, i} \Omega_{i}\right], \\
& W_{2}^{\Phi}\left(\bar{m}_{\ell i}, \tilde{\phi}\right)=\frac{1}{2}\left[\left(\bar{m}_{\ell i} \tilde{\phi} \tilde{\Phi}_{i}\right)_{, \ell}-\bar{m}_{\ell i, \ell} \Omega_{i}\right] .
\end{aligned}
$$

Evidently, $W_{2}^{\Phi}$ depends on the components of the deviatoric couple-stress tensor, which are provided through well-defined constitutive equations (Spencer and Soldatos, 2007; 
Soldatos, 2014, 2015, 2018). In contrast, $W_{1}^{\Phi}$ depends on the spherical part of the couplestress which does not influence equilibrium and, as is thus left undetermined in the Mindlin and Tiersten model, is long regarded in the literature as a quantity that remains indeterminate in the couple-stress theory.

\subsection{The spherical part of the couple-stress tensor}

It is now noted that in the special case of the Mindlin and Tiersten model, the leading assumption (4.1) converts (4.10) into the following:

$$
\begin{aligned}
& W_{1}^{\Phi}\left(m_{r r}, \tilde{\phi}\right) \equiv W_{1}^{\Omega}\left(m_{r r}, \Omega_{i}\right)=\frac{1}{6}\left[\left(m_{r r} \Omega_{i}\right)_{, i}-m_{r r, i} \Omega_{i}\right]=\frac{1}{6} m_{r r} \Omega_{i, i}=0, \\
& W_{2}^{\Phi}\left(\bar{m}_{\ell i}, \tilde{\phi}\right) \equiv W_{2}^{\Omega}\left(m_{r r}, \Omega_{i}\right)=\frac{1}{2}\left[\left(\bar{m}_{\ell i} \Omega_{i}\right)_{, \ell}-\bar{m}_{\ell i, \ell} \Omega_{i}\right]=\frac{1}{2} \bar{m}_{\ell i} \Omega_{i, \ell},
\end{aligned}
$$

where use is made of the identity $\Omega_{i, i}=0$, along with (4.7). The first of these relations holds true regardless of the value of the spherical part of the couple stress, $m_{r r}$, which thus needs not be determined. With $W_{1}^{\Omega}$ being identically zero, $W_{2}^{\Omega}$ is necessarily, and correctly, identical to the spin-gradient energy $W^{\Omega}$, which is the polar part of the strain energy function employed in the Mindlin and Tiersten model (see equation (3.3b) of part II). It is thus seen that the analysis outlined in Section 4.2 does not contradict any of the postulates, claims or conclusions associated with the Mindlin and Tiersten model.

However, unlike the direct manner that $W^{\Omega}$ is set up in the Mindlin and Tiersten version of polar linear elasticity, the present framework builds up the polar part of the strain energy function, denoted with $W^{k}$ in (Spencer and Soldatos, 2007; Soldatos, 2014, 2015, 2018; Soldatos et al., 2020), on the basis of a standard method underpinned by the theory of tensor functions representation (e.g., Spencer, 1971; Zheng, 1994). That part of the stored energy emerges through consideration of deformation effects associated with the fibre direction gradients and, necessarily, should be identical with the spin energy involved in the generalized couple-stress theory $\left(W^{\Phi} \equiv W^{k}\right)$.

Most importantly, $W^{k}$ always contains an extra energy term that offers no contribution into the couple-stress constitutive equation. Its emergence in $W^{k}$ thus leaves 
unaffected the state of equilibrium, precisely as it also happens with the spherical part of the couple-stress. The presence of that energy term is in fact observed even in the strain energy density of the non-linear theory (Spencer and Soldatos, 2007), thus showing that the outlined considerations hold true in all available versions of the corresponding theoretical framework.

The next Section thus confines attention to the existing linear versions of this framework and shows that, in all three cases available, a proper consideration of the extra energy term that enters the curvature-strain part of strain energy function enables determination of the spherical part, $m_{r r}$, of the couple-stress tensor.

\section{Determination of the spherical part of the couple-stress tensor}

As is also noted in (Soldatos et al, 2020), there have now become available three versions of polar linear elasticity for fibrous composites that exhibit transverse isotropy due to an embedded family of fibres resistant in bending. These are:

(i) the completely unrestricted version of the theory (Spencer and Soldatos, 2007; Soldatos, 2014), which considers that the symmetric and the antisymmetric parts of the curvature-strain tensor (see also (5.11) and (5.12) below) enter the strain energy function in an independent manner: this is the complete version of the theory that couples effects due to all three, fibre-bending, fibre-splay and fibretwist deformation modes and, hence, employs seven elastic moduli in the constitutive equation of the deviatoric part of the couple-stress;

(ii) a special case of the same (Soldatos et al., 2020), which considers that the symmetric and the antisymmetric parts of the curvature-strain tensor enter the strain function in a symmetric manner: this restricted version of the theory accounts for effects of the fibre-splay deformation mode only and, as it neglects effects due to fibrebending and fibre-twist, employs only two elastic moduli in the deviatoric part of the couple-stress constitutive equation;

(iii) the initial restricted version of the theory, first considered in (Spencer and Soldatos, 2007; Soldatos, 2014, 2018a), which neglects effects due to fibre-splay and fibre- 
twist: this version considers that the predominant and more influential fibre deformation mode is that of fibre-bending and thus makes use of just a single elastic modulus in the constitutive equation of the deviatoric couple-stress.

For the sake of presentation convenience, these versions of the theory are next considered and discussed in the inverse order, thus starting from the simplest and ending with the most complicated one.

\subsection{Version (iii) of the linear theory: Fibre-bending deformation mode}

The curvature-strain part of the strain energy function employed in this version of the theory is

$$
W^{K}=\frac{3}{8} d^{f} K_{j} K_{j}+\bar{\gamma}\left(a_{j} K_{j}\right)^{2}, \quad K_{i}=u_{i, k j} a_{k} a_{j},
$$

where $K_{i}$ stands for the components of the relevant fibre curvature vector (Soldatos, 2014; 2018a). Moreover, $d^{f}>0$ stands for the single fibre-bending stiffness that enters actively the constitutive equation of the deviatoric part of the couple-stress tensor, $\bar{m}_{\ell r}=d^{f} \varepsilon_{r s i} K_{i} a_{\ell} a_{s}$.

The second elastic modulus appearing in (5.1), $\bar{\gamma}>0$, is associated with the aforementioned extra energy term that leaves unaffected both the constitutive equation (5.2) and the state of equilibrium.

Introduction of the constitutive equation (5.2) and its symmetric stress counterpart into the equilibrium equations (2.3) can convert the latter into their Navier-type displacement representation. Potential solution of the latter will thus enable determination of the displacement components, $u_{i}$, and consequently of all the remaining physical quantities, with the exception the spherical part of the couple-stress, $m_{r r}$, and the parameter $\tilde{\phi}$ introduced in (4.7); see, for instance, relevant example applications detailed in (Dagher and Soldatos, 2011; Farhat and Soldatos, 2015; Soldatos et al., 2019).

Nevertheless, a comparison of (5.1) with (4.9) reveals that, necessarily, it is 


$$
\begin{aligned}
& W_{1}^{\Phi}\left(m_{r r}, \tilde{\phi}\right) \equiv \frac{1}{6}\left[\left(m_{r r} \tilde{\phi} \tilde{\Phi}_{i}\right)_{, i}-m_{r r, i} \Omega_{i}\right]=\bar{\gamma}\left(a_{j} K_{j}\right)^{2}, \\
& W_{2}^{\Phi}\left(\bar{m}_{\ell i}, \tilde{\phi}\right) \equiv \frac{1}{2}\left[\left(\bar{m}_{\ell i} \tilde{\phi} \tilde{\Phi}_{i}\right)_{, \ell}-\bar{m}_{\ell i, \ell} \Omega_{i}\right]=\frac{3}{8} d^{f} K_{j} K_{j},
\end{aligned}
$$

where use is also made of (4.10). By virtue of (4.5) and (4.6), the second of these relations may thus provide the unknown variable parameter $\tilde{\phi}$ through solution of the first order partial differential equation (PDE):

$$
\left[\left(\bar{m}_{\ell i} \tilde{\Phi}_{i}\right) \tilde{\phi}\right]_{, \ell}=\bar{m}_{\ell i, \ell} \Omega_{i}+\frac{3}{4} d^{f} K_{j} K_{j}
$$

Use of (4.7) then leads to full determination of the fibre-spin vector, $\boldsymbol{\Phi}$, and enables (5.3a) to obtain the form

$$
\left(\Phi_{i}-\Omega_{i}\right) m_{r r, i}+\Phi_{i, i} m_{r r}=6 \bar{\gamma}\left(a_{j} K_{j}\right)^{2}
$$

which is a first order PDE for the last remaining unknown, $m_{r r}$. Hence, solution of this equation, subject to boundary conditions that are consistent with any well-posed boundary value problem, enables determination of the spherical part of the couple-stress tensor.

It is thus seen that, although the additional elastic modulus appearing in (5.1), $\bar{\gamma}$, remains inactive in the constitutive as well as in the Navier-type displacement equations of this version of the theory, it does participate actively in the PDE (5.5) and, through potential solution of the latter, enables determination of the spherical part of the couplestress tensor.

As an illustrating example, consider the special case of straight fibres met often in applications (e.g., Farhat and Soldatos, 2015; Soldatos et al., 2019) and align the $x_{1}$-axis with the fibre direction. By thus assuming that $\boldsymbol{a}=(1,0,0)^{\mathrm{T}}$, the components of the fibre curvature vector (5.1) become

$$
K_{i}=u_{i, 11} \text {, }
$$

and the only non-zero components of the deviatoric couple-stress tensor (5.2) thus are

$$
\bar{m}_{12}=-d^{f} K_{3}=-d^{f} u_{3,11}, \quad \bar{m}_{13}=d^{f} K_{2}=d^{f} u_{2,11} \text {. }
$$

In turn, the components of the vector $\tilde{\boldsymbol{\Phi}}$ are found to be

$\tilde{\Phi}_{1}=0, \quad \tilde{\Phi}_{2}=-u_{3,1}, \quad \tilde{\Phi}_{3}=u_{2,1}$,

and the PDE (5.4) obtains the more specific form 
$\left[\left(u_{2,11} u_{2,1}+u_{3,11} u_{3,1}\right) \tilde{\phi}\right]_{, 1}=-u_{3,111} \Omega_{2}+u_{2,111} \Omega_{3}+\frac{3}{4}\left(u_{1,11}^{2}+u_{2,11}^{2}+u_{3,11}^{2}\right)$

where, by virtue of (2.11a) and (2.9b), the appearing components of the spin-vector $\boldsymbol{\Omega}$ are considered as known functions of the already determined displacement gradients.

Finally, as only two components of the fibre-spin vector $\boldsymbol{\Phi}=\tilde{\phi} \tilde{\boldsymbol{\Phi}}$ are non-zero, equation (5.5) obtains the following explicit form:

$-\Omega_{1} m_{r r, 1}+\left(\Phi_{2}-\Omega_{2}\right) m_{r r, 2}+\left(\Phi_{3}-\Omega_{3}\right) m_{r r, 3}+\left(\Phi_{2,2}+\Phi_{3,3}\right) m_{r r}=6 \bar{\gamma} K_{1}^{2}$.

The variable coefficients of this PDE depend on displacement gradients of several orders, as well as on the parameter $\tilde{\phi}$, which is already determined, either analytically or numerically, through solution of (5.9). Potential solution of the first order PDE (5.10) will thus provide the value of spherical part of the couple-stress.

\subsection{Version (ii): Fibre-splay deformation mode}

The curvature-strain part of the strain energy function employed in this, as well as in the unrestricted version of the theory (Section 5.3 below) makes use of the full form of the fibre curvature-strain tensor

$\kappa_{i j}=\left(u_{i, k} a_{k}\right)_{, j}=u_{i, j k} a_{k}+u_{i, k} a_{k, j}$.

That part of the strain energy function is thus of the form $W^{\kappa}\left(\boldsymbol{\kappa}_{s}, \boldsymbol{\kappa}_{a}, \mathbf{a}\right)$, where the components of the symmetric, $\boldsymbol{\kappa}_{\boldsymbol{s}}$, and the anti-symmetric, $\boldsymbol{\kappa}_{\boldsymbol{a}}$, part of $\boldsymbol{\kappa}$ are, respectively,

$$
\begin{aligned}
& \kappa_{(i j)}=\frac{1}{2}\left(\kappa_{i j}+\kappa_{j i}\right)=\frac{1}{2}\left[\left(u_{i, k} a_{k}\right)_{, j}+\left(u_{j, k} a_{k}\right)_{, i}\right], \\
& \kappa_{[i j]}=\frac{1}{2}\left(\kappa_{i j}-\kappa_{j i}\right)=\frac{1}{2}\left[\left(u_{i, k} a_{k}\right)_{, j}-\left(u_{j, k} a_{k}\right)_{, i}\right] .
\end{aligned}
$$

The fibre-splay deformation version of the theory emerged in (Soldatos et al., 2020) after imposing the restriction

$W^{\kappa}\left(\boldsymbol{\kappa}_{s}, \boldsymbol{\kappa}_{a}, \boldsymbol{a}\right)=W^{\kappa}\left(\boldsymbol{\kappa}_{a}, \boldsymbol{\kappa}_{s}, \boldsymbol{a}\right)=W^{\kappa}(\boldsymbol{\kappa}, \boldsymbol{a})$.

This restriction requires from $W^{\kappa}$ to acquire the form 


$$
\begin{aligned}
W^{k} & =\beta_{1}\left(\kappa_{(n n)}\right)^{2}+\beta_{2} \kappa_{(n n)} a_{k} \kappa_{(k m)} a_{m}+\hat{\beta}_{3}\left(a_{k} \kappa_{(k m)} a_{m}\right)^{2} \\
& =\beta_{1}\left(\kappa_{n n}\right)^{2}+\beta_{2} \kappa_{n n} a_{k} \kappa_{k m} a_{m}+\hat{\beta}_{3}\left(a_{k} \kappa_{k m} a_{m}\right)^{2}
\end{aligned}
$$

and, hence, to make use of three elastic moduli, whose values are required to obey the inequalities

$\beta_{1} \geq 0, \quad \beta_{2}+\hat{\beta}_{3} \geq 0, \quad \hat{\beta}_{3} \geq \beta_{2}^{2} / 4 \beta_{1}$.

However, only two of these elastic moduli enter actively the corresponding constitutive equation of the deviatoric part of the couple-stress,

$$
\bar{m}_{\ell r}=\frac{2}{3} \varepsilon_{r \ell s} a_{s}\left(2 \beta_{1} \kappa_{n n}+\beta_{2} \kappa_{k m} a_{k} a_{m}\right)=\frac{2}{3} \varepsilon_{r \ell s} a_{s}\left(2 \beta_{1} \kappa_{(n n)}+\beta_{2} \kappa_{(k m)} a_{k} a_{m}\right) .
$$

The spherical part of the couple-stress can then be determined in the manner detailed in the previous Section, through appropriate use of the term of $W^{k}$ that involves the relevant inactive modulus, $\hat{\beta}_{3}$.

Accordingly, a comparison of (5.14) with (4.9) reveals that

$$
\begin{aligned}
& W_{1}^{\Phi}\left(m_{r r}, \tilde{\phi}\right) \equiv \frac{1}{6}\left[\left(m_{r r} \tilde{\phi} \tilde{\Phi}_{i}\right)_{, i}-m_{r r, i} \Omega_{i}\right]=\hat{\beta}_{3}\left(a_{k} \kappa_{k m} a_{m}\right)^{2}, \\
& W_{2}^{\Phi}\left(\bar{m}_{\ell i}, \tilde{\phi}\right) \equiv \frac{1}{2}\left[\left(\bar{m}_{\ell i} \tilde{\phi} \tilde{\Phi}_{i}\right)_{, \ell}-\bar{m}_{\ell i, \ell} \Omega_{i}\right]=\beta_{1}\left(\kappa_{n n}\right)^{2}+\beta_{2} \kappa_{n n} a_{k} \kappa_{k m} a_{m} .
\end{aligned}
$$

The second of these relations will then provide the variable parameter $\tilde{\phi}$ as solution of the first order PDE

$$
\left[\left(\bar{m}_{\ell i} \tilde{\Phi}_{i}\right) \tilde{\phi}\right]_{, \ell}=\bar{m}_{\ell i, \ell} \Omega_{i}+2 \beta_{1}\left(\kappa_{n n}\right)^{2}+2 \beta_{2} \kappa_{n n} a_{k} \kappa_{k m} a_{m} .
$$

As (4.7) thus enables determination $\boldsymbol{\Phi},(5.17 \mathrm{a})$ obtains the form of the first order PDE

$$
\left(\Phi_{i}-\Omega_{i}\right) m_{r r, i}+\Phi_{i, i} m_{r r}=6 \hat{\beta}_{3}\left(a_{k} \kappa_{k m} a_{m}\right)^{2},
$$

whose solution enables determination of the last remaining unknown, $m_{r r}$.

In the special case of straight fibres, where $\boldsymbol{a}=(1,0,0)^{\mathrm{T}}$, the components of the curvature-strain tensor (5.11) become $\kappa_{i j}=u_{i, j 1}$,

and the non-zero components of the deviatoric couple-stress tensor (5.16) are

$$
\bar{m}_{23}=-\bar{m}_{32}=\frac{2}{3}\left(2 \beta_{1}+\beta_{2}\right) e_{11,1}+\frac{4}{3} \beta_{1}\left(e_{22,1}+e_{33,1}\right) \text {. }
$$


As is also pointed out in (Soldatos et al., 2020), this result reveals in a comprehensive manner that, unlike its fibre-bending mode counterpart, the present version of the theory makes no use of spin-gradient kinematic variables and, hence, is entirely incompatible with the anisotropic version of the polar linear elasticity model due to Mindlin and Tiersten.

Indeed, this restricted version of the linear theory discards the spin-gradients employed by Cosserat and Cosserat (1909) as well as by Mindlin and Tiersten (1962) and, instead, employs the indicated strain-gradients as principal kinematic variables. By undermining the importance of both the fibre-bending and the fibre-twist deformation modes, it thus confines attention to polar material effects which are predominantly associated with the fibre-splay types of deformation.

It is recalled that Part II noted with interest the presence of both spin- and straingradients among the principal kinematic variables of the unrestricted version of the present theoretical framework. However, no attempt was made in Part II towards either interpretation of the physical meaning and significance of the observed strain-gradient variables or identification of their nature and source of origin. In this regard, the generalised formulation of the couple-stress theory presented earlier in Sections 2 and 3 enables next a complete clarification of these issues.

5.3 Version (i) - Unrestricted theory: Full coupling of fibre-bending, -splay and -twist deformation modes

In this case, the curvature part of the strain energy function is still an objective function of the symmetric and antisymmetric parts (5.12) of the curvature-strain tensor but is neither restricted by the condition (5.13) nor in any other way (Spencer and Soldatos, 2007; Soldatos, 2014). Its general form is accordingly as follows (see also (Soldatos et al., 2020)):

$$
W^{\kappa}=\beta_{1} J_{1}^{2}+\beta_{2} J_{1} J_{2}+\hat{\beta}_{3} J_{2}^{2}+\beta_{3} J_{3}+\beta_{4} J_{4}+\beta_{5} J_{5}+\beta_{6} J_{6}+\beta_{7} J_{7},
$$

where the appearing deformation invariants are

$J_{1}=\operatorname{tr} \boldsymbol{\kappa}_{s}=\operatorname{tr} \boldsymbol{\kappa}, J_{2}=\mathbf{a} \boldsymbol{\kappa}_{s} \mathbf{a}=\mathbf{a} \boldsymbol{\kappa} \mathbf{a}, J_{3}=\operatorname{tr} \boldsymbol{\kappa}_{s}^{2}, J_{4}=\mathbf{a} \boldsymbol{\kappa}_{s}^{2} \mathbf{a}, J_{5}=\operatorname{tr} \boldsymbol{\kappa}_{a}^{2}, J_{6}=\mathbf{a} \boldsymbol{\kappa}_{a}^{2} \mathbf{a}, J_{7}=\mathbf{a} \boldsymbol{\kappa}_{s} \boldsymbol{\kappa}_{a} \mathbf{a}$.

It is observed that the first three of the appearing, eight in total coefficients correspond to their counterparts employed earlier in (5.14). 
This observation makes immediately understood the reason that $\hat{\beta}_{3}$ is again inactive in the corresponding couple-stress constitutive equation,

$$
\begin{aligned}
\bar{m}_{\ell r}= & \frac{2}{3} \varepsilon_{r \ell s} a_{s}\left(2 \beta_{1} \kappa_{n n}+\beta_{2} \kappa_{k m} a_{k} a_{m}\right)+\frac{2}{3} \varepsilon_{r i s} a_{s}\left(2 \beta_{3} \kappa_{(i \ell)}+\beta_{4} \kappa_{(i n)} a_{n} a_{\ell}\right)- \\
& \frac{1}{3} \varepsilon_{r i s}\left\{4 \beta_{5}\left(a_{s} \kappa_{[i \ell]}+a_{\ell} \kappa_{[i s]}\right)-2 \beta_{6} a_{n} a_{\ell}\left(a_{i} \kappa_{[s n]}-2 a_{s} \kappa_{[i n]}\right)+\beta_{7} a_{n} a_{\ell}\left(a_{i} \kappa_{n s}-2 a_{s} \kappa_{i n}\right)\right\} .
\end{aligned}
$$

More information regarding the range of values of the coefficients appearing in (5.22) and (5.24) is given in (Soldatos, 2014; Soldatos et al., 2020) and is not repeated here. Nevertheless, as $\hat{\beta}_{3}$ is not involved in (5.24), the spherical part of the couple-stress tensor can again be determined in the manner detailed in the previous pair of Sections.

Accordingly, a comparison of (5.22) with (4.9) reveals that

$$
\begin{aligned}
& W_{1}^{\Phi}\left(m_{r r}, \tilde{\phi}\right) \equiv \frac{1}{6}\left[\left(m_{r r} \tilde{\phi} \tilde{\Phi}_{i}\right)_{, i}-m_{r r, i} \Omega_{i}\right]=\hat{\beta}_{3} J_{2}^{2}, \\
& W_{2}^{\Phi}\left(\bar{m}_{\ell i}, \tilde{\phi}\right) \equiv \frac{1}{2}\left[\left(\bar{m}_{\ell i} \tilde{\phi} \tilde{\Phi}_{i}\right)_{, \ell}-\bar{m}_{\ell i, \ell} \Omega_{i}\right]=\beta_{1} J_{1}^{2}+\beta_{2} J_{1} J_{2}+\beta_{3} J_{3}+\beta_{4} J_{4}+\beta_{5} J_{5}+\beta_{6} J_{6}+\beta_{7} J_{7} .
\end{aligned}
$$

The first of these relations is identical to (5.17a) and can be used in the same manner for the determination of $m_{r r}$, as soon as $\tilde{\phi}$ is determined through solution of the first order PDE (5.25b). The first two terms appearing in the right-hand side of (5.25b) are identical to their (5.17b) counterparts. Here, however, these are accompanied by five additional terms which thus enable the effects of the fibre-splay deformation mode encountered in the preceding Section to couple with their fibre-bending and -twist deformation mode counterparts.

The consequences of the outlined revelations become again easier understood in the special case of straight fibres, where $\boldsymbol{a}=(1,0,0)^{T}$. In that case, the nonzero components of the deviatoric couple-stress tensor are given by the following constitutive equations (Spencer and Soldatos, 2007; Soldatos, 2018b): 


$$
\begin{aligned}
& {\left[\begin{array}{c}
\bar{m}_{11} \\
\bar{m}_{22} \\
\bar{m}_{33}
\end{array}\right]=\left[\begin{array}{rr}
0 & 2 b_{3} \\
-b_{2} & b_{3} \\
b_{2} & b_{3}
\end{array}\right]\left[\begin{array}{l}
e_{23,1} \\
\Omega_{1,1}
\end{array}\right],} \\
& {\left[\begin{array}{c}
-\bar{m}_{32} \\
\bar{m}_{23} \\
-\bar{m}_{12} \\
\bar{m}_{13}
\end{array}\right]=\left[\begin{array}{ccccccc}
d_{11} & d_{22} & d_{33} & 0 & 0 & 0 & 0 \\
d_{11} & d_{33} & d_{22} & 0 & 0 & 0 & 0 \\
0 & 0 & 0 & d_{23} & d_{32} & 0 & 0 \\
0 & 0 & 0 & 0 & 0 & d_{23} & -d_{32}
\end{array}\right]\left[\begin{array}{l}
e_{11,1} \\
e_{22,1} \\
e_{33,1} \\
e_{31,1} \\
\Omega_{2,1} \\
e_{12,1} \\
\Omega_{3,1}
\end{array}\right],}
\end{aligned}
$$

where, the appearing elastic moduli relate to their counterparts involved in (5.22) as follows:

$$
\begin{aligned}
& b_{2}=\frac{4}{3} \beta_{3}, b_{3}=\frac{4}{3} \beta_{5}, d_{11}=\frac{2}{3}\left(2 \beta_{1}+\beta_{2}\right), d_{22}=\frac{4}{3}\left(\beta_{1}+\beta_{3}\right), d_{33}=\frac{4}{3} \beta_{1}, \\
& d_{23}=\frac{1}{3}\left(4 \beta_{3}+2 \beta_{4}+3 \beta_{7}\right), d_{32}=\frac{1}{3}\left(12 \beta_{5}+6 \beta_{6}-2 \beta_{4}-\beta_{7}\right) .
\end{aligned}
$$

It is seen that, unlike either of its restricted versions, the unrestricted theory predicts that the normal components of deviatoric couple-stress are generally non-zero. However, in line with the conventional couple-stress theory postulation (Cosserat and Cosserat, 1909), the relevant part of the constitutive equations, namely (5.26a), leaves still undetermined the spherical part of the couple-stress tensor. As noted with interest in Part II though, the spingradients appearing in (5.26) are accompanied by additional kinematic variables of the form $e_{i j, 1}$, and these make the unrestricted theory incompatible with the conventional couple-stress theory (Cosserat and Cosserat, 1909) and its Mindlin and Tiersten (1962) version.

As already mentioned, further comparisons between the two theories were not attempted in Part II where, however, it was already noted that the curvature-strain part of the strain energy function can be decomposed in two parts as follows:

$W^{K}=W^{\Omega}+W^{E}$,

where,

$W^{\Omega}=D_{55} \Omega_{1,1}^{2}+D_{77}\left(\Omega_{2,1}^{2}+\Omega_{3,1}^{2}\right)$

is a quadratic function of the spin-gradients only, while 
$W^{E}=\left(e_{11,1}, e_{22,1}, e_{33,1}\right)\left[\begin{array}{ccc}D_{11} & D_{12} & D_{12} \\ D_{12} & D_{22} & D_{23} \\ D_{12} & D_{23} & D_{22}\end{array}\right]\left(\begin{array}{l}e_{11,1} \\ e_{22,1} \\ e_{33,1}\end{array}\right)+D_{44} e_{23,1}^{2}+D_{66}\left(e_{31,1}^{2}+e_{12,1}^{2}\right)$

is a quadratic function of the strain-gradients, and

$$
\begin{aligned}
& D_{11}=\beta_{1}+\beta_{2}+\beta_{3}+\beta_{4}+\hat{\beta}_{3}, \quad D_{12}=\beta_{1}+\beta_{2} / 2, \quad D_{23}=\beta_{1}, \quad D_{22}=\beta_{1}+\beta_{3}, \\
& D_{44}=2 \beta_{3}, \quad D_{55}=-2 \beta_{5}, \quad D_{66}=2 \beta_{3}+\beta_{4}, \quad D_{77}=-\left(2 \beta_{5}+\beta_{6}\right) .
\end{aligned}
$$

The new relevant discoveries, made afterwards in (Soldatos et al, 2020) as well as in the present investigation, lead now to the conclusion that (i) the strain-gradient kinematic variables involved in $W^{E}$ include effects of fibre-splay deformation nature, and (ii) such effects cannot be captured by the Mindlin and Tiersten model, whose polar elasticity part is based on spin-gradient kinematic variables only.

In view of these observations, it is finally seen that, in the case of straight fibres, equations (5.25) obtain the simplified form:

$$
\begin{aligned}
W_{1}^{\Phi} & \equiv \frac{1}{6}\left[\left(m_{r r} \tilde{\phi} \tilde{\Phi}_{i}\right)_{, i}-m_{r r, i} \Omega_{i}\right]=\hat{\beta}_{3} u_{1,11}^{2}, \\
W_{2}^{\Phi} & \equiv \frac{1}{2}\left[\left(\bar{m}_{\ell i} \tilde{\phi} \tilde{\Phi}_{i}\right)_{, \ell}-\bar{m}_{\ell i, \ell} \Omega_{i}\right]=W^{\Omega}+\tilde{W}^{E},
\end{aligned}
$$

and, as is detailed already, their solution will determine the value of the spherical part, $m_{r r}$ of the couple-stress tensor. The couple-stress tensor will thus become fully determined, by superposing the thus obtained value of $m_{r r}$ on the constitutive equation $(5.26 \mathrm{a})$, in the manner shown in (2.4).

\section{Conclusions}

As is also concluded in Soldatos et al. (2020), the Mindlin and Tiersten (1962) polar linear elasticity model fails, in general, to agree with its (Spencer and Soldatos, 2007; Soldatos, 2014) counterpart, which makes use of a set of additional kinematic variables. These emerge in the latter model in the form of strain-gradients and furnish the model with ability to couple together effects due to all three of the observed fibre-bending, fibre-splay and fibre-twist deformation modes. This revelation leads also to the conclusion that, despite its generality, 
the degree of anisotropy involved in the Mindlin and Tiersten model is not able to capture effects of fibre-splay type of deformation.

The present investigation identified as reason of the observed theoretical disagreement the fact that the characteristic spin-vector that measures fibre deformation is not necessarily identical with its counterpart employed in conventional couple-stress theory (Cosserat and Cosserat, 1909; Mindlin and Tiersten, 1962); namely, the vector of the antisymmetric rotation tensor met in non-polar linear elasticity. The new, generalised formulation of the couple-stress theory presented in Section 2 thus makes a proper distinction between those two different spin-vectors, and succeeds to create room for the newly emerged, fibre-splay type kinematic variables to enter and, hence, be accounted for. Most interestingly, all theorems associated in Part II (Soldatos, 2018b) with the Mindlin and Tiersten (1962) model are essentially still valid, subject to slight, almost marginal modification.

Moreover, the present generalised formulation provides a convincing answer to a long-standing question regarding the indeterminacy of the spherical part of the couple-stress tensor, at least as far as polar elasticity of fibre-reinforced materials is concerned. Indeed, the generalised couple-stress theory still agrees that the spherical part of the couple-stress remains indeterminate in the special case of the Mindlin and Tiersten model. However, in the case of fibrous composites with embedded fibres resistant in bending, it provides additional means, and an extra piece of information that enable full determination of the couple-stress tensor, including its spherical part. In doing so, it requires from that extra piece of information to suitably accommodate the fact that the Spencer and Soldatos (2007) theoretical framework introduces an extra energy term that offers no contribution to the constitutive equations and, like the spherical part of the couple-stress, does not influence the state of equilibrium.

The outlined revelations were exemplified in the special case of fibrous composites reinforced by a single family of straight fibres. Namely, a case considered already in several stress analysis applications (Dagher and Soldatos, 2011; Farhat and Soldatos, 2015; Soldatos et al., 2019). It should be noted in this regard, that the results and discussions described, as well as conclusions made in those stress analysis studies are still valid and are not directly affected by the here presented revelations. 
This is because determination of the newly introduced, characteristic fibre-spin deformation vector and, subsequently, of the spherical part of the couple-stress, become possible only after the state of equilibrium is fully studied in any well-posed boundary value problem and, hence, only after the relevant solution is achieved of the Navier-type displacement equations. As the latter equations are already solved in each of the implied boundary value problems (Dagher and Soldatos, 2011; Farhat and Soldatos, 2015; Soldatos et al., 2019), the obtained solution is now directly employable for the determination of both the relevant fibre-spin vector and the associated spherical part of the couple-stress tensor.

\section{References}

1. Cosserat, E. and Cosserat, F., 1909. Théorie des Corps Deformables. Hermann, Paris, 1909.

2. Dagher, M.A. and Soldatos, K.P., 2011. On small azimuthal shear deformation of fibrereinforced cylindrical tubes. J. Mech. Mater. Struct. 6, 141-168.

3. Eringen, A.C., 1968. Theory of micropolar elasticity. In: Liebowitz, H. (ed.) Fracture, vol. 2, pp. 621-729. Academic Press, New York.

4. Farhat, A.F. and Soldatos, K.P., 2015. Cylindrical bending and vibration of polar material laminates. Mech. Adv. Mater. Struct. 22, 885-896.

5. Hadjesfandiari, A.R., Dargush, G.F, 2011. Couple stress theory for solids. Int. J. Solids Struct. 48, 24962510.

6. Jones, R.M., 1996. Mechanics of Composite Materials, Taylor \& Francis, Washington.

7. Koiter, W.T., 1964. Couple stresses in the theory of elasticity, I and II. Proc. Ned. Akad. Wet. Ser B. 67, 17-44.

8. Mindlin, R.D. and Tiersten, H.F., 1962. Effects of couple-stresses in linear elasticity, Arch. Ration. Mech. Anal. 11, 415-448.

9. Mindlin, R.D. and Eshel, E.E., 1964.On first strain-gradient theories in linear elasticity, Int. J, Solids Struct. 4, 109-124.

10. Spencer, A.J.M., 1971. Theory of invariants. In: Eringen, A.C. (ed.) Continuum Physics, vol. 1, pp. 239-253. Academic Press, New York. 
11. Spencer, A.J.M. and Soldatos, K.P., 2007. Finite deformations of fibrereinforced elastic solids with fibre bending stiffness. Int. J. Non-lin. Mech. 42, 355-368.

12. Soldatos, K.P., 2014. Foundation of polar linear elasticity for fibre-reinforced materials. J. Elast. 114, 155-178.

13. Soldatos, K.P., 2015. Foundation of polar linear elasticity for fibre-reinforced materials II: Advanced anisotropy. J. Elasticity 118, 223-242.

14. Soldatos, K.P., 2018a. On the characterisation of fibrous composites when fibres resist bending. Int. J. Solids Struct. 146-149.

15. Soldatos, K.P., 2018b. On the characterisation of fibrous composites when fibres resist bending - Part II: Connection with anisotropic polar linear elasticity. Int. J. Solids Struct. 152-153, 1-11.

16. Soldatos, K.P., Aydogdu, M. and Gul, U., 2019. Plane strain polar elasticity of fibre reinforced functionally graded materials and structures. J. Mech. Mater. Struct. 14, 497535.

17. Soldatos, K.P., Shariff, M.H.B.M. and Merodio, J., 2020. On the constitution of polar fibre-reinforced materials. Mech. Adv. Meter. Struct., ISSN: 1537-6494 (Print) 15376532 (Online) DOI: 10.1080/15376494.2020.1729449.

18. Ting, T.C.T., 1996. Anisotropic Elasticity. Oxford University Press, New York.

19. Truesdell, C. and Toupin, R.A., 1960. The Classical Field Theories. Encycl. Phys. III/1, 226-793, Springer-Verlag, Berlin.

20. Zheng, Q.S., 1994. Theory of representations for tensor functions: A unified invariant approach to constitutive equations. Appl. Mech. Rev. 47, 545-587. 